\title{
Elliptic Function Solutions in Jackiw-Teitelboim Dilaton Gravity
}

\author{
Jennie D'Ambroise ${ }^{1}$ and Floyd L. Williams ${ }^{2}$ \\ ${ }^{1}$ Department of Mathematics/CIS, SUNY Old Westbury, P.O. Box 210, Old Westbury, NY 11568, USA \\ ${ }^{2}$ Department of Mathematics and Statistics, University of Massachusetts, Amherst, MA 01003, USA
}

Correspondence should be addressed to Jennie D’Ambroise; jdambroise@gmail.com

Received 24 April 2017; Accepted 14 May 2017; Published 31 July 2017

Academic Editor: Andrei D. Mironov

Copyright (C) 2017 Jennie D’Ambroise and Floyd L. Williams. This is an open access article distributed under the Creative Commons Attribution License, which permits unrestricted use, distribution, and reproduction in any medium, provided the original work is properly cited.

\begin{abstract}
We present a new family of solutions for the Jackiw-Teitelboim model of two-dimensional gravity with a negative cosmological constant. Here, a metric of constant Ricci scalar curvature is constructed, and explicit linearly independent solutions of the corresponding dilaton field equations are determined. The metric is transformed to a black hole metric, and the dilaton solutions are expressed in terms of Jacobi elliptic functions. Using these solutions, we compute, for example, Killing vectors for the metric.
\end{abstract}

\section{Introduction}

It is well known that the Einstein gravitational field equations for a vacuum (with a zero matter tensor) are automatically solved by any metric $g$ on a two-dimensional space-time $M$. A proof of this fact is given in Section 2 of [1], for example. A nontrivial theory of gravity for such an $M$ was worked out in 1984 by Jackiw and Teitelboim (J-T). This involves in addition to $g$ a scalar field $\Phi$ on $M$ called a dilaton field; see $[2,3]$. The pair $(g, \Phi)$ is subject to the equations of motion

$$
\begin{gathered}
R(g)=\frac{2}{l^{2}}, \\
\nabla_{i} \nabla_{j} \Phi=\frac{g_{i j} \Phi}{l^{2}}
\end{gathered}
$$

derived from the action integral

$$
S(g, \Phi)=\text { constant } \cdot \int_{M} d^{2} x \sqrt{|\operatorname{det} g|} \Phi\left(R(g)-\frac{2}{l^{2}}\right)
$$

where $R(g)$ is the constant Ricci scalar curvature of $g$ and the (negative) cosmological constant is $\Lambda=-1 / l^{2}$. In local coordinates $\left(x_{1}, x_{2}\right)$ on $M$, the Hessian in (1) is given by

$$
\nabla_{i} \nabla_{j} \Phi=\frac{\partial^{2} \Phi}{\partial x_{i} \partial x_{j}}-\sum_{k=1}^{2} \Gamma_{i j}^{k} \frac{\partial \Phi}{\partial x_{k}}, \quad 1 \leq i, j \leq 2
$$

where $\Gamma_{i j}^{k}$ are the Christoffel symbols (of the second kind) of $g$ [1]. The J-T theory has, for example, the (Lorentzian) black hole solution

$$
g: d s^{2}=-\left(m^{2} r^{2}-M\right) d T^{2}+\frac{d r^{2}}{m^{2} r^{2}-M},
$$

with coordinates $\left(x_{1}, x_{2}\right)=(T, r)$, where

$$
\begin{aligned}
\Lambda & =-m^{2}, \\
R(g) & =2 m^{2}, \\
\Phi(T, r) & \stackrel{\text { def. }}{=} m r,
\end{aligned}
$$

with $M$ being a black hole mass parameter. Here and throughout, we note that our sign convention for scalar curvature is the negative of that used in $[2,3]$ and by others in the literature.

The purpose of this paper is the following. For real numbers $a, b \neq 0$ and for a soliton velocity parameter $v$, we consider the following metric in the variables $\left(x_{1}, x_{2}\right)=$ $(\tau, \rho)$ :

$$
\begin{aligned}
d s^{2} & \stackrel{\text { def. }}{=} a^{2} b^{2} \mathrm{dn}^{2}(\rho, \kappa)\left[\left(\frac{a^{2} \kappa^{4} \operatorname{sn}^{2}(\rho, \kappa) \mathrm{cn}^{2}(\rho, \kappa)}{\operatorname{dn}^{2}(\rho, \kappa)}\right.\right. \\
& \left.-\frac{v^{2}}{4}\right) d \tau^{2}
\end{aligned}
$$




$$
\begin{aligned}
& -\frac{\kappa^{4} \operatorname{sn}^{2}(\rho, \kappa) \mathrm{cn}^{2}(\rho, \kappa)}{\operatorname{dn}^{2}(\rho, \kappa)}\left(\frac{a^{2} \kappa^{4} \operatorname{sn}^{2}(\rho, \kappa) \mathrm{cn}^{2}(\rho, \kappa)}{\operatorname{dn}^{2}(\rho, \kappa)}\right. \\
& \left.\left.-\frac{v^{2}}{4}\right)^{-1} d \rho^{2}\right],
\end{aligned}
$$

where $\operatorname{sn}(x, \kappa), \operatorname{cn}(x, \kappa)$, and $\operatorname{dn}(x, \kappa)$ are the standard Jacobi elliptic functions with modulus $\kappa ; 0 \leq \kappa \leq 1$ [4]. We will generally assume that

$$
\left|\frac{v}{2 a \kappa^{2}}\right|>1, \quad \kappa \neq 0
$$

As will be seen later, this metric is the diagonalization of a metric constructed from solutions $r(x, t), s(x, t)$ of the reaction diffusion system

$$
\begin{aligned}
& r_{t}-r_{x x}+\frac{2}{b^{2}} r^{2} s=0, \\
& s_{t}+s_{x x}-\frac{2}{b^{2}} r s^{2}=0 .
\end{aligned}
$$

We will explicate the solutions $r(x, t), s(x, t)$ in terms of the elliptic function $\operatorname{dn}(x, \kappa)$. Remarkably, the metric in (6) has constant scalar curvature $R(g)=8 / b^{2}$ so that the first equation in (1) holds. The main work of the paper then is to solve the corresponding system of partial differential equations (the dilaton field equations) in (1), which for $g$ in (6) are

$$
\nabla_{i} \nabla_{j} \Phi=\frac{R(g)}{2} g_{i j} \Phi=\frac{4}{b^{2}} g_{i j} \Phi, \quad 1 \leq i, j \leq 2
$$

Here the cosmological constant is $\Lambda=-4 / b^{2}$.

Given the complicated nature of our $g$, system (9) is necessarily quite difficult to solve directly. Our method is to construct a series of transformations of variables so that $g$ in (6) is transformed to $g$ in (4). Then we can use the simple solution $\Phi(T, r)=m r$ in (5) and other known solutions to work backwards through these transformations of variables to construct $\Phi(\tau, \rho)$ that satisfies (9). The various details involved, with further remarks that lead to (6), will be the business of Sections 2, 3, and 4.

In the end, we obtain the following main result: the metric in (6) solves the first J-T equation of motion (1). Namely, $R(g)=8 / b^{2}$, as we have remarked. Also three linearly independent solutions of the field equations in (1), namely, of the system of equations (9), are given by

$$
\begin{gathered}
\Phi^{(1)}(\tau, \rho)=2 a^{2} \operatorname{dn}^{2}(\rho, \kappa)+\frac{v^{2}}{4}-a^{2}\left(2-\kappa^{2}\right), \\
\Phi^{(2)}(\tau, \rho)=\operatorname{dn}(\rho, \kappa) \sinh (\sqrt{A} \tau) \\
\cdot \sqrt{\frac{v^{2}}{4}-\frac{a^{2} \kappa^{4} \operatorname{sn}^{2}(\rho, \kappa) \mathrm{cn}^{2}(\rho, \kappa)}{\operatorname{dn}^{2}(\rho, \kappa)}}
\end{gathered}
$$

$$
\begin{gathered}
\Phi^{(3)}(\tau, \rho)=\operatorname{dn}(\rho, \kappa) \cosh (\sqrt{A} \tau) \\
\cdot \sqrt{\frac{v^{2}}{4}-\frac{a^{2} \kappa^{4} \operatorname{sn}^{2}(\rho, \kappa) \mathrm{cn}^{2}(\rho, \kappa)}{\operatorname{dn}^{2}(\rho, \kappa)}}
\end{gathered}
$$

for

$$
A \stackrel{\text { def. }}{=} \frac{v^{4}}{16}-\frac{v^{2} a^{2}}{2}\left(2-\kappa^{2}\right)+a^{4} \kappa^{4},
$$

which we assume is nonzero. Given (7), we shall see in Section 4 that $A=0$ only for $a= \pm\left(1-\sqrt{1-\kappa^{2}}\right) v / 2 \kappa^{2}$ and moreover that the second expression under the radical (i.e., $\left.v^{2} / 4-\cdots\right)$ in (10) is positive. For $\kappa=1, A=\left(v^{2} / 4-a^{2}\right)^{2}>0$, but we can have $A<0$ for some $\kappa<1$. Also for $\kappa=1$ the solutions in (10) reduce to those given in (60), with (6) given by (61).

\section{Reaction Diffusion Systems and Derivation of the Metric in (6)}

Since metric (6) is one of the main objects of interest, we indicate in this section its derivation. For a constant $B$, consider the system of partial differential equations

$$
\begin{aligned}
& r_{t}-r_{x x}+B r^{2} s=0, \\
& s_{t}+s_{x x}-B r s^{2}=0
\end{aligned}
$$

in the variables $(x, t)$. This system is a special case of the more general reaction diffusion system (RDS),

$$
\begin{aligned}
& r_{t}=d_{r} r_{x x}+F(r, s), \\
& s_{t}=d_{s} s_{x x}+G(r, s),
\end{aligned}
$$

which occurs in chemistry, physics, or biology, for example, where $d_{r}$ and $d_{s}$ are diffusion constants and $F$ and $G$ are growth and interaction functions. The key point for us is that from solutions $r(x, t)$ and $s(x, t)$ of (12) one can construct a metric $g$ of constant Ricci scalar curvature $R(g)=4 B$ by the following prescription [5-7]:

$$
\begin{aligned}
d s^{2} \stackrel{\text { def. }}{=} g_{11} d t^{2}+2 g_{12} d t d x+g_{22} d x^{2}, \\
\quad g_{11} \stackrel{\text { def. }}{=}-r_{x} s_{x}, g_{12} \stackrel{\text { def. }}{=} \frac{1}{2}\left(s r_{x}-r s_{x}\right), g_{22} \stackrel{\text { def. }}{=} r s .
\end{aligned}
$$

One could also simply start with the definitions in (14), apart from the preceding references that employ Cartan's zweibein formalism [8], and use a Maple program (tensor), for example, to check directly that indeed $R(g)=4 B$. Our 
interest is in the choice $B=2 / b^{2}$, where, for real $a, b$, and $v$, with $a, b \neq 0$ as in Section $1, r(x, t), s(x, t)$ given by

$$
\begin{aligned}
& r(x, t) \stackrel{\text { def. }}{=} a b \operatorname{dn}(a(x-v t), \kappa) \\
& \cdot \exp \left(\left[\frac{v^{2}}{4}+a^{2}\left(2-\kappa^{2}\right)\right] t-\frac{v x}{2}\right), \\
& s(x, t) \stackrel{\text { def. }}{=}-a b \operatorname{dn}(a(x-v t), \kappa) \\
& \cdot \exp \left(-\left[\frac{v^{2}}{4}+a^{2}\left(2-\kappa^{2}\right)\right] t+\frac{v x}{2}\right)
\end{aligned}
$$

are solutions of system (12), which also could be checked directly by Maple. For $B=2 / b^{2}$, (12) is system (8) with solutions (15) promised in Section 1, and $g$ in (14) has the scalar curvature $4 B=8 / b^{2}$ discussed in Section 1 . From [4], various formulas like

$$
\begin{aligned}
\operatorname{sn}^{2}(x, \kappa)+\operatorname{cn}^{2}(x, \kappa) & =1, \\
\operatorname{dn}^{2}(x, \kappa)+\kappa^{2} \operatorname{sn}^{2}(x, \kappa) & =1, \\
\frac{d}{d x} \operatorname{sn}(x, \kappa) & =\operatorname{cn}(x, \kappa) \operatorname{dn}(x, \kappa), \\
\frac{d}{d x} \operatorname{cn}(x, \kappa) & =-\operatorname{sn}(x, \kappa) \operatorname{dn}(x, \kappa), \\
\frac{d}{d x} \operatorname{dn}(x, \kappa) & =-\kappa^{2} \operatorname{sn}(x, \kappa) \operatorname{cn}(x, \kappa)
\end{aligned}
$$

are available. Using prescription (14), one computes that

$$
\begin{aligned}
g_{11} & =a^{2} b^{2}\left[a^{2} \kappa^{4} \operatorname{sn}^{2}(a(x-v t), \kappa) \mathrm{cn}^{2}(a(x-v t), \kappa)\right. \\
& \left.-\frac{v^{2}}{4} \mathrm{dn}^{2}(a(x-v t), \kappa)\right], \\
g_{12} & =\frac{a^{2} b^{2} v}{2} \operatorname{dn}^{2}(a(x-v t), \kappa), \\
g_{22} & =-a^{2} b^{2} \operatorname{dn}^{2}(a(x-v t), \kappa) .
\end{aligned}
$$

For $\rho \stackrel{\text { def. }}{=} a(x-v t)$, so that $d \rho=a(d x-v d t), g$ can be expressed more conveniently as

$$
\begin{aligned}
d s^{2} & =a^{2} b^{2} \operatorname{dn}^{2}(\rho, \kappa) \\
\cdot & {\left[\left(\frac{a^{2} \kappa^{4} \operatorname{sn}^{2}(\rho, \kappa) \mathrm{cn}^{2}(\rho, \kappa)}{\operatorname{dn}^{2}(\rho, \kappa)}-\frac{v^{2}}{4}\right) d t^{2}-\frac{v}{a} d t d \rho\right.} \\
& \left.-\frac{d \rho^{2}}{a^{2}}\right] .
\end{aligned}
$$

The goal now is to set up a change of variables $(t, \rho) \rightarrow$ $(\tau, \rho)$ so that $g$ in $(18)$ is transformed to $(6)$, where the cross term $d \tau d \rho$ does not appear, in comparison with the term $d t d \rho$ appearing in (18). For this purpose note first, in general, that for

$$
h=A(\rho) d t^{2}+C_{1}(\rho) d \rho d t+C_{2}(\rho) d \rho^{2}
$$

the change of variables $\tau=t+\phi(\rho)$ gives $d t=d \tau-\phi^{\prime}(\rho) d \rho$ and $d t^{2}=d \tau^{2}-2 \phi^{\prime}(\rho) d \tau d \rho+\phi^{\prime}(\rho)^{2} d \rho^{2}$ and

$$
\begin{aligned}
h= & A(\rho) d \tau^{2}+\left[-2 \phi^{\prime}(\rho) A(\rho)+C_{1}(\rho)\right] d \tau d \rho \\
& +\left[A(\rho) \phi^{\prime}(\rho)^{2}-C_{1}(\rho) \phi^{\prime}(\rho)+C_{2}(\rho)\right] d \rho^{2} .
\end{aligned}
$$

The condition that the cross term $d \tau d \rho$ does not appear is therefore that $\phi(\rho)$ satisfies

$$
\phi^{\prime}(\rho)=\frac{C_{1}(\rho)}{2 A(\rho)} .
$$

Apply this to (18):

$$
\phi^{\prime}(\rho)=\frac{-v}{2 a} \cdot\left(\frac{a^{2} \kappa^{4} \operatorname{sn}^{2}(\rho, \kappa) \mathrm{cn}^{2}(\rho, \kappa)}{\operatorname{dn}^{2}(\rho, \kappa)}-\frac{v^{2}}{4}\right)^{-1} .
$$

Now, by (16), $\mathrm{dn}^{2}(x, \kappa)=1-\kappa^{2} \operatorname{sn}^{2}(x, \kappa)=\operatorname{sn}^{2}(x, \kappa)+$ $\mathrm{cn}^{2}(x, \kappa)-\kappa^{2} \operatorname{sn}^{2}(x, \kappa)=\mathrm{cn}^{2}(x, \kappa)+\left(1-\kappa^{2}\right) \operatorname{sn}^{2}(x, \kappa) \geq$ $\mathrm{cn}^{2}(x, \kappa)$ and $\operatorname{sn}^{2}(x, \kappa) \leq \operatorname{sn}^{2}(x, \kappa)+\operatorname{cn}^{2}(x, \kappa)=1 \Rightarrow$ $\operatorname{sn}^{2}(x, \kappa) \operatorname{cn}^{2}(x, \kappa) / \operatorname{dn}^{2}(x, \kappa) \leq 1$. If the term in parenthesis in (22) was zero, this would therefore force the inequality $v^{2} / 4 a^{2} \kappa^{4} \leq 1$. That is, if $\left|v / 2 a \kappa^{2}\right|>1$, which is the assumption in (7), then $v^{2} / 4 a^{2} \kappa^{4}>1$ and therefore the denominator term in parenthesis in (22) is nonzero, which means that $\phi^{\prime}(\rho)$ is a continuous function and (22) therefore indeed has a solution $\phi(\rho)$, with assumption (7) imposed. Also, the coefficient of $d \rho^{2}$ in $(20)$ is

$$
\begin{gathered}
a^{2} b^{2} \operatorname{dn}^{2} Q \frac{v^{2}}{4 a^{2} Q^{2}}-a b^{2} v \operatorname{dn}^{2} \frac{v}{2 a Q}-b^{2} \mathrm{dn}^{2} \\
=-\frac{b^{2} v^{2} \mathrm{dn}^{2}}{4 Q}-b^{2} \mathrm{dn}^{2}
\end{gathered}
$$

where for convenience we write sn, $\mathrm{cn}$, and $\operatorname{dn}$ for $\operatorname{sn}(\rho, \kappa)$, $\operatorname{cn}(\rho, \kappa), \operatorname{dn}(\rho, \kappa)$ and $Q$ for

$$
Q(\rho, \kappa) \stackrel{\text { def. }}{=} \frac{a^{2} \kappa^{4} \operatorname{sn}^{2}(\rho, \kappa) \mathrm{cn}^{2}(\rho, \kappa)}{\operatorname{dn}^{2}(\rho, \kappa)}-\frac{v^{2}}{4} .
$$

Then

$$
\begin{aligned}
Q+\frac{v^{2}}{4} & =\frac{a^{2} \kappa^{4} \mathrm{sn}^{2} \mathrm{cn}^{2}}{\mathrm{dn}^{2}} \Longrightarrow \\
1+\frac{v^{2}}{4 Q} & =\frac{a^{2} \kappa^{4} \mathrm{sn}^{2} \mathrm{cn}^{2}}{\mathrm{dn}^{2} Q} \Longrightarrow \\
-b^{2} \mathrm{dn}^{2}-\frac{b^{2} v^{2} \mathrm{dn}^{2}}{4 Q} & =-\frac{a^{2} b^{2} \kappa^{4} \mathrm{sn}^{2} \mathrm{cn}^{2}}{Q}
\end{aligned}
$$


which is the coefficient of $d \rho^{2}$ in (20) by (23). Then, by (21), (20) reads

$$
h=a^{2} b^{2} \mathrm{dn}^{2} \mathrm{Q} d \tau^{2}-\frac{a^{2} b^{2} \kappa^{4} \mathrm{sn}^{2} \mathrm{cn}^{2}}{Q} d \rho^{2},
$$

which is (6). That is, we have verified that the change of variables $\tau=t+\phi(\rho)$ with $\phi(\rho)$ subject to condition (22) (which in fact makes $\phi^{\prime}(\rho)$ a continuous function, again assuming (7)) transforms the reaction diffusion metric in (18) to the diagonal metric in (6).

In the special case when the elliptic modulus $\kappa=1$,

$$
\begin{aligned}
& \operatorname{sn}(x, 1)=\tanh (x), \\
& \operatorname{cn}(x, 1)=\operatorname{dn}(x, 1)=\operatorname{sech}(x)
\end{aligned}
$$

and (18) and (6) simplify:

$$
\begin{aligned}
d s^{2} & =a^{2} b^{2} \operatorname{sech}^{2} \rho\left[\left(a^{2} \tanh ^{2} \rho-\frac{v^{2}}{4}\right) d t^{2}-\frac{v}{a} d t d \rho\right. \\
& \left.-\frac{d \rho^{2}}{a^{2}}\right], \\
d s^{2} & =a^{2} b^{2} \operatorname{sech}^{2} \rho\left[\left(a^{2} \tanh ^{2} \rho-\frac{v^{2}}{4}\right) d t^{2}\right. \\
& \left.-\tanh ^{2} \rho\left(a^{2} \tanh ^{2} \rho-\frac{v^{2}}{4}\right)^{-1} d \rho^{2}\right],
\end{aligned}
$$

which are the line elements (3.12) and (3.14), respectively, in [6]; $a$ here corresponds to the notation $k$ there. Also the cosmological constant $\Lambda_{0}$ in [6] corresponds to our $2 \Lambda=$ $-8 / b^{2}: b^{2}=8 /\left(-\Lambda_{0}\right)$. Similarly, $r$ and $s$ in (15) reduce to the dissipative soliton solutions $q^{+}$and $q^{-}$, respectively, in (2.32) of [6], apart from the factor $b$. One can also explicitly determine $\phi(\rho)$ in $(22)$.

\section{Transformation of the Metric in (6) to a J-T Black Hole Metric}

Now that the existence of the metric in (6) has been described in the context of a reaction diffusion system (namely, (8)), the strategy of this section is to set up a series of changes of variables, as indicated in the introduction, which transforms it to the simpler J-T form (4). Other applications, of independent interest, can flow from this, apart from our main focus to solve system (9). A general method to go from (6) to (4) has been developed by the first named author. Alternatively, one can generalize part of the argument in [6] which leads at least to a Schwarzschild form, as we do here, and then argue a bit more to obtain the J-T form, with the final result being expressed by (39)-(41) below.

Start with the change of variables $r=|a| \operatorname{dn}(\rho, \kappa)$ so that $d r=-\kappa^{2}|a| \operatorname{sn}(\rho, \kappa) \cdot \operatorname{cn}(\rho, \kappa) d \rho$ by $(16) \Rightarrow$

$$
\frac{d r^{2}}{r^{2}}=\frac{\kappa^{4} \operatorname{sn}^{2}(\rho, \kappa) \mathrm{cn}^{2}(\rho, \kappa) d \rho^{2}}{\operatorname{dn}^{2}(\rho, \kappa)} .
$$

Also by (16),

$$
\begin{aligned}
& \frac{a^{2} \kappa^{4} \operatorname{sn}^{2}(\rho, \kappa) \mathrm{cn}^{2}(\rho, \kappa)}{\operatorname{dn}^{2}(\rho, \kappa)} \\
& =\frac{a^{2} \kappa^{2}\left(1-\operatorname{dn}^{2}(\rho, \kappa)\right)\left(1-\operatorname{sn}^{2}(\rho, \kappa)\right)}{\operatorname{dn}^{2}(\rho, \kappa)} \\
& =\frac{\left(a^{2}-a^{2} \operatorname{dn}^{2}(\rho, \kappa)\right)\left(\kappa^{2}-\kappa^{2} \operatorname{sn}^{2}(\rho, \kappa)\right)}{\operatorname{dn}^{2}(\rho, \kappa)} \\
& =\frac{\left(a^{2}-r^{2}\right)\left(\kappa^{2}+\operatorname{dn}^{2}(\rho, \kappa)-1\right)}{r^{2} / a^{2}} \\
& =\frac{\left(a^{2}-r^{2}\right)\left(\kappa^{2}-1+r^{2} / a^{2}\right)}{r^{2} / a^{2}} \\
& =\frac{\left(a^{2}-r^{2}\right)}{r^{2}}\left[a^{2}\left(\kappa^{2}-1\right)+r^{2}\right] \Longrightarrow \\
& \frac{a^{2} \kappa^{4} \operatorname{sn}^{2}(\rho, \kappa) \mathrm{cn}^{2}(\rho, \kappa)}{\operatorname{dn}^{2}(\rho, \kappa)}-\frac{v^{2}}{4} \\
& =\frac{\left(a^{2}-r^{2}\right)}{r^{2}}\left[a^{2}\left(\kappa^{2}-1\right)+r^{2}\right]-\frac{v^{2}}{4} \\
& =\frac{a^{4}\left(\kappa^{2}-1\right)}{r^{2}}+2 a^{2}-a^{2} \kappa^{2}-r^{2}-\frac{v^{2}}{4} \\
& =\frac{a^{4}\left(\kappa^{2}-1\right)}{r^{2}}+2 a^{2}-a^{2} \kappa^{2}-r^{2}-r_{0}^{2}-a^{2} \kappa^{4}
\end{aligned}
$$

for

$$
r_{0}^{2} \stackrel{\text { def. }}{=} \frac{v^{2}}{4}-a^{2} \kappa^{4}>0
$$

Again by (7), $v^{2} / 4 a^{2} \kappa^{4}>1 \Rightarrow v^{2} / 4>a^{2} \kappa^{4} \Rightarrow$ indeed $r_{0}^{2}>0$. By (29) and (30), we see that we can write (6) as

$$
\begin{aligned}
g & =b^{2}\left[-r^{2}\left(r^{2}+r_{0}^{2}+a^{2}\left(\kappa^{4}+\kappa^{2}-2\right)\right.\right. \\
& \left.+\frac{a^{4}\left(1-\kappa^{2}\right)}{r^{2}}\right) d \tau^{2}+\left(r^{2}+r_{0}^{2}\right. \\
& \left.\left.+a^{2}\left(\kappa^{4}+\kappa^{2}-2\right)+\frac{a^{4}\left(1-\kappa^{2}\right)}{r^{2}}\right)^{-1} d r^{2}\right] .
\end{aligned}
$$

Next let $x \stackrel{\text { def. }}{=}\left(2 r^{2}+r_{0}^{2}\right) / r_{0}^{4}$, as in (3.18) of [6], but where our $r_{0}^{2}$ in (31) generalizes their $r_{0}^{2}$, and for convenience let

$$
\begin{aligned}
& \alpha \stackrel{\text { def. }}{=} a^{2}\left(\kappa^{4}+\kappa^{2}-2\right), \\
& \beta \stackrel{\text { def. }}{=} a^{4}\left(1-\kappa^{2}\right)
\end{aligned}
$$


in (32). Then $g$ in (32) assumes the form

$$
\begin{aligned}
g & =b^{2}\left[\left(-\frac{r_{0}^{4}}{4}\left(r_{0}^{4} x^{2}-1\right)-\frac{\alpha r_{0}^{2}}{2}\left(r_{0}^{2} x-1\right)-\beta\right) d \tau^{2}\right. \\
& +\frac{1}{16} \\
& \left.\cdot r_{0}^{8}\left(\frac{r_{0}^{4}}{4}\left(r_{0}^{4} x^{2}-1\right)+\frac{\alpha}{2} r_{0}^{2}\left(r_{0}^{2} x-1\right)+\beta\right)^{-1} d x^{2}\right] \\
& =\frac{b^{2} r_{0}^{4}}{4}\left[-\left(r_{0}^{4} x^{2}-1+\frac{2 \alpha}{r_{0}^{2}}\left(r_{0}^{2} x-1\right)+\frac{4 \beta}{r_{0}^{4}}\right) d \tau^{2}\right. \\
& \left.+\left(r_{0}^{4} x^{2}-1+\frac{2 \alpha}{r_{0}^{2}}\left(r_{0}^{2} x-1\right)+\frac{4 \beta}{r_{0}^{4}}\right)^{-1} d x^{2}\right]
\end{aligned}
$$

which generalizes the Schwarzschild form (3.19) of [6], since for $\kappa=1$ we have that $\alpha=\beta=0$ in (33).

For the change of variables $t=A_{0} \tau$ and $r_{-}=A_{0} x$ with $A_{0} \stackrel{\text { def. }}{=}|b| r_{0}^{2} / 2$, the Schwarzschild $g$ in (34) goes to

$$
\begin{aligned}
g= & -\left[\frac{4}{b^{2}} r_{-}^{2}-1+\frac{2 \alpha}{r_{0}^{2}}\left(\frac{2}{|b|} r_{-}-1\right)+\frac{4 \beta}{r_{0}^{4}}\right] d t^{2} \\
& +\left[\frac{4}{b^{2}} r_{-}^{2}-1+\frac{2 \alpha}{r_{0}^{2}}\left(\frac{2}{|b|} r_{-}-1\right)+\frac{4 \beta}{r_{0}^{4}}\right]^{-1} d r_{-}^{2},
\end{aligned}
$$

which in turn goes to

$$
\begin{aligned}
g= & -\left[\frac{4}{b^{2}} r_{1}^{2}-b^{2}+\frac{2 \alpha}{r_{0}^{2}}\left(\frac{2 b}{|b|} r_{1}-b^{2}\right)+\frac{4 \beta b^{2}}{r_{0}^{4}}\right] d T^{2} \\
& +\left[\frac{4 r_{1}^{2}}{b^{2}}-b^{2}+\frac{2 \alpha}{r_{0}^{2}}\left(\frac{2 b}{|b|} r_{1}-b^{2}\right)+\frac{4 \beta b^{2}}{r_{0}^{4}}\right]^{-1} d r_{1}^{2}
\end{aligned}
$$

by way of the change of variables $t=b T$ and $r_{-}=r_{1} / b$. We need one final observation: in general a metric of the form

$$
\begin{aligned}
g_{1}= & -\left[A_{1} x^{2}+B_{1} x+C_{1}\right] d T^{2} \\
& +\left[A_{1} x^{2}+B_{1} x+C_{1}\right]^{-1} d x^{2},
\end{aligned}
$$

say $A_{1} \neq 0$, can be transformed to the J-T form (4); namely,

$$
\begin{aligned}
g_{1}= & -\left[A_{1} r^{2}+C_{1}-\frac{B_{1}^{2}}{4 A_{1}}\right] d T^{2} \\
& +\left[A_{1} r^{2}+C_{1}-\frac{B_{1}^{2}}{4 A_{1}}\right]^{-1} d r^{2},
\end{aligned}
$$

by way of the change of variables $r=x+B_{1} / 2 A_{1}$. Apply this to (36) with $x$ playing the role of $r_{1}$ there:

$$
\begin{aligned}
g= & -\left[A_{1} r^{2}+C_{1}-\frac{B_{1}^{2}}{4 A_{1}}\right] d T^{2} \\
& +\left[A_{1} r^{2}+C_{1}-\frac{B_{1}^{2}}{4 A_{1}}\right]^{-1} d r^{2}
\end{aligned}
$$

for

$$
\begin{aligned}
& A_{1} \stackrel{\text { def. }}{=} \frac{4}{b^{2}}, \\
& B_{1} \stackrel{\text { def. }}{=} \frac{4 \alpha b}{r_{0}^{2}|b|}, \\
& C_{1} \stackrel{\text { def. }}{=}-b^{2}-\frac{2 \alpha b^{2}}{r_{0}^{2}}+\frac{4 \beta b^{2}}{r_{0}^{4}} .
\end{aligned}
$$

Using definition (33) for $\alpha, \beta$ and $r_{0}^{2}=v^{2} / 4-a^{2} \kappa^{4}$, which is definition (31), one computes that

$$
C_{1}-\frac{B_{1}^{2}}{4 A_{1}}=\frac{-b^{2}}{r_{0}^{4}}\left[\frac{v^{4}}{16}-\frac{a^{2} v^{2}}{2}\left(2-\kappa^{2}\right)+a^{4} \kappa^{4}\right]
$$

in (39).

\section{Derivation of Solutions (10) of the Field Equations (9)}

The main result is derived in this section. Namely, we indicate how the series of changes of variables in Section 3 (according to remarks in Introduction) lead to the linearly independent solutions $\Phi^{(j)}(\tau, \rho), j=1,2,3$, in (10) of the dilaton field equations in (9). There the metric elements $g_{i j}$ are given by (6). For $Q(\rho, \kappa)$ in $(24)$,

$$
\begin{aligned}
& g_{11} \stackrel{\text { def. }}{=} a^{2} b^{2} \operatorname{dn}^{2}(\rho, \kappa) Q(\rho, \kappa), \\
& g_{12}=g_{21}=0, \\
& g_{22}=a^{2} b^{2} \operatorname{dn}^{2}(\rho, \kappa)\left(\frac{-\kappa^{4} \operatorname{sn}^{2}(\rho, \kappa) \mathrm{cn}^{2}(\rho, \kappa)}{\operatorname{dn}^{2}(\rho, \kappa)}\right) \\
& \cdot Q(\rho, \kappa)^{-1},
\end{aligned}
$$

and $\nabla_{i} \nabla_{j} \Phi$ are given by (3) for $\left(x_{1}, x_{2}\right)=(\tau, \rho)$. The Christoffel symbols $\Gamma_{i j}^{k}$ in (3) (which could be computed, e.g., by Maple) will not be needed for the derivation of (10), although they could be used to verify these solutions. Obviously any dilaton solution could be replaced by any nonzero multiple of itself. In the following then, we can disregard such multiples if we wish to.

In addition to the dilaton solution $\Phi^{(1)}(T, r) \stackrel{\text { def. }}{=} m r$ in (5) for metric (4) in the variables $(T, r)$, there are solutions

$$
\begin{aligned}
& \Phi^{(2)}(T, r) \stackrel{\text { def. }}{=} \sqrt{m^{2} r^{2}-M} \sinh (m \sqrt{M} T), \\
& \Phi^{(3)}(T, r) \stackrel{\text { def. }}{=} \sqrt{m^{2} r^{2}-M} \cosh (m \sqrt{M} T) .
\end{aligned}
$$

We work backwards the changes of variables in Section 3 for $\Phi^{(1)}(T, r)$ and $\Phi^{(2)}(T, r)$, for example, to see how one arrives at the first two solutions $\Phi^{(1)}(\tau, \rho)$ and $\Phi^{(2)}(\tau, \rho)$ in $(10)$ in the variables $(\tau, \rho)$. 
Starting with the (39) version of (4), we have $m^{2}=A_{1}=$ $4 / b^{2}$ by (40), with $M=-\left(C_{1}-B_{1}^{2} / 4 A_{1}\right)$ given by (41). Here $m \sqrt{M}=\sqrt{B_{1}^{2}-4 A_{1} C_{1}} / 2($ for $m=2 /|b|) \Rightarrow$

$$
\begin{aligned}
& \Phi^{(1)}(T, r)=\frac{2}{|b|} r, \\
& \Phi^{(2)}(T, r) \\
& \quad=\sqrt{A_{1} r^{2}+C_{1}-\frac{B_{1}^{2}}{4 A_{1}}} \sinh \left(\frac{\sqrt{B_{1}^{2}-4 A_{1} C_{1}}}{2} T\right) .
\end{aligned}
$$

By the final change of variables $r=r_{1}+B_{1} / 2 A_{1}$ in Section 3, we see that $A_{1} r^{2}=A_{1} r_{1}^{2}+B_{1} r_{1}+B_{1}^{2} / 4 A_{1} \Rightarrow$

$$
\begin{aligned}
& \Phi^{(1)}\left(T, r_{1}\right)=\frac{2}{|b|}\left(r_{1}+\frac{B_{1}}{2 A_{1}}\right), \\
& \Phi^{(2)}\left(T, r_{1}\right) \\
& \quad=\sqrt{A_{1} r_{1}^{2}+B_{1} r_{1}+C_{1}} \sinh \left(\frac{\sqrt{B_{1}^{2}-4 A_{1} C_{1}}}{2} T\right) .
\end{aligned}
$$

The change of variables $t=b T$ and $r_{-}=r_{1} / b$ preceded the change $r=r_{1}+B_{1} / 2 A_{1}$, so that

$$
\begin{aligned}
& \Phi^{(1)}\left(t, r_{-}\right)=\frac{2 b}{|b|} r_{-}+\frac{B_{1}}{|b| A_{1}}, \\
& \Phi^{(2)}\left(t, r_{-}\right) \\
& \quad=\sqrt{4 r_{-}^{2}+B_{1} b r_{-}+C_{1}} \sinh \left(\frac{\sqrt{B_{1}^{2}-4 A_{1} C_{1}}}{2 b} t\right),
\end{aligned}
$$

since $A_{1} b^{2} \stackrel{\text { def. }}{=} 4$. We had $t=A_{0} \tau$ and $r_{-}=A_{0} x$ for $A_{0} \stackrel{\text { def. }}{=}$ $|b| r_{0}^{2} / 2$, which gives

$$
\begin{aligned}
& \Phi^{(1)}(\tau, x)=b r_{0}^{2} x+\frac{B_{1}}{|b| A_{1}}, \\
& \frac{|b|}{b} \Phi^{(1)}(\tau, x)=|b| r_{0}^{2} x+\frac{B_{1}}{b A_{1}}, \\
& \Phi^{(2)}(\tau, x)=\sqrt{b^{2} r_{0}^{4} x^{2}+\frac{B_{1} b|b|}{2} r_{0}^{2} x+C_{1}} \\
& \cdot \sinh \left(\frac{\sqrt{B_{1}^{2}-4 A_{1} C_{1}}}{4} \cdot \frac{|b| r_{0}^{2}}{b} \tau\right),
\end{aligned}
$$

for the Schwarzschild version of our metric in (34). Next let $x=\left(2 r^{2}+r_{0}^{2}\right) / r_{0}^{4}$ to get

$$
\begin{aligned}
& \Phi^{(1)}(\tau, r)=\frac{|b|\left(2 r^{2}+r_{0}^{2}\right)}{r_{0}^{2}}+\frac{B_{1}}{b A_{1}}, \\
& \Phi^{(2)}(\tau, r) \\
& =\sqrt{\frac{b^{2}\left(2 r^{2}+r_{0}^{2}\right)^{2}}{r_{0}^{4}}+\frac{B_{1} b|b|}{2} \frac{\left(2 r^{2}+r_{0}^{2}\right)}{r_{0}^{2}}+C_{1}} \\
& \cdot \sinh \left(\frac{\sqrt{B_{1}^{2}-4 A_{1} C_{1}}}{4} r_{0}^{2} \tau\right),
\end{aligned}
$$

where we have disregarded the multiple $|b| / b= \pm 1$ in (47) and have used $\sinh (|b| x / b)=(|b| / b) \sinh (x)$. Finally, the first change of variables $r=|a| \operatorname{dn}(\rho, \kappa)$ in Section 3 gives

$$
\Phi^{(1)}(\tau, \rho)=\frac{|b|}{r_{0}^{2}}\left(2 a^{2} \operatorname{dn}^{2}(\rho, \kappa)+r_{0}^{2}\right)+\frac{\alpha|b|}{r_{0}^{2}}
$$

by definition (40). If we disregard the multiple $|b| / r_{0}^{2}$ in (49) and use $r_{0}^{2}+\alpha \stackrel{\text { def. }}{=} v^{2} / 4+a^{2}\left(\kappa^{2}-2\right)$ by definitions (31) and (33), we obtain from (49) the first solution

$$
\Phi^{(1)}(\tau, \rho)=2 a^{2} \operatorname{dn}^{2}(\rho, \kappa)+\frac{v^{2}}{4}+a^{2}\left(\kappa^{2}-2\right)
$$

in (10). More work is required of course to obtain the second solution there.

First, we note that, by (40) and (41),

$$
\begin{aligned}
& B_{1}^{2}-4 A_{1} C_{1}=-4 A_{1}\left(C_{1}-\frac{B_{1}^{2}}{4 A_{1}}\right) \\
& =\frac{16}{r_{0}^{4}}\left[\frac{v^{4}}{16}-\frac{a^{2} v^{2}}{2}\left(2-\kappa^{2}\right)+a^{4} \kappa^{4}\right] \Longrightarrow \\
& \frac{\sqrt{B_{1}^{2}-4 A_{1} C_{1}}}{4} r_{0}^{2} \tau=\sqrt{\frac{v^{4}}{16}-\frac{a^{2} v^{2}}{2}\left(2-\kappa^{2}\right)+a^{4} \kappa^{4} \cdot \tau,}
\end{aligned}
$$

which is $\sqrt{A} \tau$ in (10). Also, for $r=|a| \operatorname{dn}, \operatorname{dn}=\operatorname{dn}(\rho, \kappa)$, the quantity under the other radical in (48) is

$$
\begin{aligned}
\frac{b^{2}}{r_{0}^{4}}\left(2 a^{2} \mathrm{dn}^{2}+r_{0}^{2}\right)^{2}+\frac{B_{1} b|b|}{2 r_{0}^{2}}\left(2 a^{2} \mathrm{dn}^{2}+r_{0}^{2}\right)+C_{1} \\
=\frac{4 a^{4} b^{2}}{r_{0}^{4}} \mathrm{dn}^{4}+\left(\frac{4 a^{2} b^{2}}{r_{0}^{2}}+\frac{B_{1} b|b| a^{2}}{r_{0}^{2}}\right) \mathrm{dn}^{2}+b^{2} \\
+\frac{B_{1} b|b|}{2}+C_{1},
\end{aligned}
$$


where, by definition (40),

$$
\begin{aligned}
& \frac{B_{1} b|b| a^{2}}{r_{0}^{2}}=\frac{4 a^{2} b^{2} \alpha}{r_{0}^{4}} \\
& b^{2}+\frac{B_{1} b|b|}{2}+C_{1} \\
& =b^{2}+\frac{2 b^{2} \alpha}{r_{0}^{2}}-b^{2}-\frac{2 \alpha b^{2}}{r_{0}^{2}}+\frac{4 \beta b^{2}}{r_{0}^{4}}=\frac{4 \beta b^{2}}{r_{0}^{4}} \Longrightarrow \\
& \frac{4 a^{2} b^{2}}{r_{0}^{2}}+\frac{B_{1} b|b| a^{2}}{r_{0}^{2}}=\frac{4 r_{0}^{2} a^{2} b^{2}+4 a^{2} b^{2} \alpha}{r_{0}^{4}} \\
& =\frac{4 a^{2} b^{2}}{r_{0}^{4}}\left(r_{0}^{2}+\alpha\right)=\frac{4 a^{2} b^{2}}{r_{0}^{4}}\left[\frac{v^{2}}{4}+a^{2}\left(\kappa^{2}-2\right)\right]
\end{aligned}
$$

again by definitions (31) and (33). That is, since $\beta=a^{4}\left(1-\kappa^{2}\right)$ by definition (33), the quantity in (52) (which is under the radical in (48) for $r=|a| \mathrm{dn})$ is

$$
\begin{aligned}
& \frac{4 a^{4} b^{2}}{r_{0}^{4}} \operatorname{dn}^{4}+\frac{4 a^{2} b^{2}}{r_{0}^{4}}\left[\frac{v^{2}}{4}+a^{2}\left(\kappa^{2}-2\right)\right] \mathrm{dn}^{2} \\
& +\frac{4 a^{4} b^{2}\left(1-\kappa^{2}\right)}{r_{0}^{4}}=\frac{4 a^{2} b^{2}}{r_{0}^{4}}\left[a^{2} \operatorname{dn}^{4}\right. \\
& \left.+\left(\frac{v^{2}}{4}+a^{2}\left(\kappa^{2}-2\right)\right) \mathrm{dn}^{2}+a^{2}\left(1-\kappa^{2}\right)\right] .
\end{aligned}
$$

We let $B(\rho)$ denote the latter bracket here. By (48), (51), and (54), we see that (for now) $\Phi^{(2)}(\tau, \rho)=\sqrt{B(\rho)} \sinh (\sqrt{A} \tau)$, if we disregard the multiple $\sqrt{4 a^{2} b^{2} / r_{0}^{4}}=2|a||b| / r_{0}^{2}$.

We find an alternate expression for $B(\rho)$, which is simpler and shows that $B(\rho)>0$, given (7). Again we write sn, $c n$, and $\operatorname{dn}$ for $\operatorname{sn}(\rho, \kappa), \operatorname{cn}(\rho, \kappa), \operatorname{dn}(\rho, \kappa)$, and we make use of (16).

$$
\begin{aligned}
& B(\rho) \stackrel{\text { def. }}{=} \mathrm{dn}^{2}\left[a^{2} \mathrm{dn}^{2}+\frac{v^{2}}{4}-2 a^{2}+a^{2} \kappa^{2}\right. \\
& \left.+a^{2} \frac{\left(1-\kappa^{2}\right)}{\mathrm{dn}^{2}}\right]=\mathrm{dn}^{2}\left[a^{2}\left(1-\kappa^{2} \mathrm{sn}^{2}\right)+\frac{v^{2}}{4}-2 a^{2}\right. \\
& \left.+a^{2} \kappa^{2}+a^{2} \frac{\left(1-\kappa^{2}\right)}{\mathrm{dn}^{2}}\right]=\mathrm{dn}^{2}\left[-a^{2} \kappa^{2} \operatorname{sn}^{2}+\frac{v^{2}}{4}\right. \\
& \left.-a^{2}\left(1-\kappa^{2}\right)+a^{2} \frac{\left(1-\kappa^{2}\right)}{\mathrm{dn}^{2}}\right]=\mathrm{dn}^{2}\left[\frac{v^{2}}{4}\right. \\
& \left.-a^{2} \kappa^{2} \operatorname{sn}^{2}+a^{2} \frac{\left(1-\kappa^{2}\right)}{\operatorname{dn}^{2}}\left(1-\mathrm{dn}^{2}\right)\right]=\operatorname{dn}^{2}\left[\frac{v^{2}}{4}\right.
\end{aligned}
$$

$$
\begin{aligned}
& \left.-a^{2} \kappa^{2} \mathrm{sn}^{2}+a^{2} \frac{\left(1-\kappa^{2}\right)}{\mathrm{dn}^{2}} \kappa^{2} \mathrm{sn}^{2}\right]=\mathrm{dn}^{2}\left[\frac{v^{2}}{4}\right. \\
& \left.-a^{2} \kappa^{2} \operatorname{sn}^{2} \frac{\left(\mathrm{dn}^{2}-1+\kappa^{2}\right)}{\mathrm{dn}^{2}}\right]=\mathrm{dn}^{2}\left[\frac{v^{2}}{4}\right. \\
& \left.-\frac{a^{2} \kappa^{2} \mathrm{sn}^{2}}{\mathrm{dn}^{2}}\left(-\kappa^{2} \mathrm{sn}^{2}+\kappa^{2}\right)\right]=\mathrm{dn}^{2}\left[\frac{v^{2}}{4}\right. \\
& \left.-\frac{a^{2} \kappa^{4} \mathrm{sn}^{2}}{\mathrm{dn}^{2}}\left(1-\mathrm{sn}^{2}\right)\right]=\mathrm{dn}^{2}\left[\frac{v^{2}}{4}-\frac{a^{2} \kappa^{4} \mathrm{sn}^{2} \mathrm{cn}^{2}}{\mathrm{dn}^{2}}\right],
\end{aligned}
$$

where we noted in Section 2 that $\mathrm{sn}^{2} \mathrm{cn}^{2} / \mathrm{dn}^{2} \leq 1$. Hence

$$
\frac{v^{2}}{4}-a^{2} \kappa^{4} \frac{\mathrm{sn}^{2} \mathrm{cn}^{2}}{\mathrm{dn}^{2}} \geq \frac{v^{2}}{4}-a^{2} \kappa^{4}>0
$$

by (7), again as in (31), and we see that $B(\rho)>0$, since $\operatorname{dn}(\rho, \kappa) \neq 0$ for $\rho$ being a real number. Moreover, we have established the desired expression for $\Phi^{(2)}(\tau, \rho)$ in $(10)$. Clearly one can replace the hyperbolic sine in the preceding discussion by the hyperbolic cosine in (43) to obtain the third solution $\Phi^{(3)}(\tau, \rho)$ in (10). To finish other claims made in Section 1 , we check that in (11) $A=0$ only for $a= \pm(1-$ $\left.\sqrt{1-\kappa^{2}}\right) v / 2 \kappa^{2}$. We continue to assume (7) of course.

The quartic equation $A=0$ has roots $a= \pm(1+$ $\left.\sqrt{1-\kappa^{2}}\right) v / 2 \kappa^{2}$ and $\pm\left(1-\sqrt{1-\kappa^{2}}\right) v / 2 \kappa^{2}$ with $a^{2}=\left(2-\kappa^{2}+\right.$ $\left.2 \sqrt{1-\kappa^{2}}\right) v^{2} / 4 \kappa^{4}$ and $\left(2-\kappa^{2}-2 \sqrt{1-\kappa^{2}}\right) v^{2} / 4 \kappa^{4}$, respectively. (7) requires that $a^{2}<v^{2} / 4 \kappa^{4}$, which forces the inequalities

$$
\begin{aligned}
& 2-\kappa^{2}+2 \sqrt{1-\kappa^{2}}<1, \\
& 2-\kappa^{2}-2 \sqrt{1-\kappa^{2}}<1,
\end{aligned}
$$

of which the first one reads $1-\kappa^{2}+2 \sqrt{1-\kappa^{2}}<0$, with the lefthand side here being $\geq 0$, a contradiction. That is, we cannot have $a= \pm\left(1+\sqrt{1-\kappa^{2}}\right) v / 2 \kappa^{2}$ which means that $a= \pm(1-$ $\left.\sqrt{1-\kappa^{2}}\right) v / 2 \kappa^{2}$. Also we check that the solutions are linearly independent: assume for constants $c_{1}, c_{2}, c_{3}$ that

$$
c_{1} \Phi^{(1)}(\tau, \rho)+c_{2} \Phi^{(2)}(\tau, \rho)+c_{3} \Phi^{(3)}(\tau, \rho)=0 .
$$

Differentiate this equation with respect to $\tau$ and evaluate the result at $(\tau, 0)$ :

$$
\frac{c_{2} \sqrt{A} \cosh (\sqrt{A} \tau)|v|}{2}+\frac{c_{3} \sqrt{A} \sinh (\sqrt{A} \tau)|v|}{2}=0
$$

since $\operatorname{dn}(0, \kappa)=1$ and $\operatorname{sn}(0, \kappa)=0$. The choice $\tau=0$ then gives $c_{2}=0$, since $A, v \neq 0$, and differentiation of the equation $c_{3} \sqrt{A} \sinh (\sqrt{A} \tau)|v| / 2=0$ and at $\tau=0$ gives $c_{3}=0$. Using again $\operatorname{dn}(0, \kappa)=1$ we see by $(10)$ that $\Phi^{(1)}(\tau, 0) \stackrel{\text { def. }}{=} v^{2} / 4+$ $a^{2} \kappa^{2}>0$ and hence also $c_{1}=0$.

Note that if $v=a=2$ and $\kappa=1 / 2$, for example, then even though $v / 2 a \kappa^{2}=2>1$ (so that (7) is satisfied), we have that $A=-12<0$. 
Again in the special case when the elliptic modulus $\kappa=1$, we have in (11) that $A=\left(v^{2} / 4-a^{2}\right)^{2}>0$ and $\sqrt{A}=v^{2} / 4-a^{2}>0$ (by (7) or (31)), and $B(\rho)=$ $\operatorname{sech}^{2} \rho\left[v^{2} / 4-a^{2} \tanh ^{2} \rho\right]=\operatorname{sech}^{2} \rho\left[v^{2}-4 a^{2} \tanh ^{2} \rho\right] / 4$; by (27), (55) $\Rightarrow \sqrt{B(\rho)}=(1 / 2) \operatorname{sech} \rho \sqrt{v^{2}-4 a^{2} \tanh ^{2} \rho}$. Here (directly) $\tanh ^{2} \rho \leq 1 \Rightarrow v^{2}-4 a^{2} \tanh ^{2} \rho>0$, again as $v^{2}>4 a^{2}$. Thus, by (10) and (28),

$$
\begin{aligned}
& \Phi^{(1)}(\tau, \rho)=2 a^{2} \operatorname{sech}^{2} \rho+\frac{v^{2}}{4}-a^{2}, \\
& \Phi^{(2)}(\tau, \rho) \\
& \quad=\left(\sinh \left(\frac{v^{2}}{4}-a^{2}\right) \tau\right)(\operatorname{sech} \rho) \sqrt{v^{2}-4 a^{2} \tanh ^{2} \rho}, \\
& \Phi^{(3)}(\tau, \rho) \\
& \quad=\left(\cosh \left(\frac{v^{2}}{4}-a^{2}\right) \tau\right)(\operatorname{sech} \rho) \sqrt{v^{2}-4 a^{2} \tanh ^{2} \rho}
\end{aligned}
$$

(where we have disregarded the multiple $1 / 2$ in $\sqrt{B(\rho)}$ ) are dilaton field solutions for the metric

$$
\begin{array}{r}
d s^{2}=a^{2} b^{2} \operatorname{sech}^{2} \rho\left[\left(a^{2} \tanh ^{2} \rho-\frac{v^{2}}{4}\right) d \tau^{2}\right. \\
\left.-\left(\tanh ^{2} \rho\right)\left(a^{2} \tanh ^{2} \rho-\frac{v^{2}}{4}\right)^{-1} d \rho^{2}\right] .
\end{array}
$$

The solutions in (60) are also new.

\section{Killing Vector Fields for Solutions (6) and (10)}

Recall that a smooth vector field $Y$ on an $n$-dimensional Riemannian manifold $(M, g)$ is called a Killing vector field (or an infinitesimal motion of $M$ ) if, for arbitrary smooth vector fields $X, Z$ on $M$,

$$
Y g(X, Z)=g([Y, X], Z)+g(X,[Y, Z])=0 .
$$

If $Y=\sum_{i=1}^{n} Y_{i}\left(\partial / \partial x_{i}\right)$ is an expression of $Y$ in terms of local coordinates $\left(x_{1}, \ldots, x_{n}\right)$ on $M$, then (62) is equivalent to the system of equations

$$
\sum_{i=1}^{n} g_{k i} \frac{\partial Y_{i}}{\partial x_{j}}+g_{j i} \frac{\partial Y_{i}}{\partial x_{k}}+\frac{\partial g_{j k}}{\partial x_{i}} Y_{i}=0
$$

for $1 \leq j, k \leq n[8,9]$. In the special (diagonal) case with $g_{i j}=0$ for $i \neq j$ and with $n=2$, the Killing equations (63) simplify to the following three equations:

$$
\begin{array}{r}
2 g_{11} \frac{\partial Y_{1}}{\partial x_{1}}+\frac{\partial g_{11}}{\partial x_{1}} Y_{1}+\frac{\partial g_{11}}{\partial x_{2}} Y_{2}=0, \\
g_{11} \frac{\partial Y_{1}}{\partial x_{2}}+g_{22} \frac{\partial Y_{2}}{\partial x_{1}}=0, \\
\frac{\partial g_{22}}{\partial x_{1}} Y_{1}+2 g_{22} \frac{\partial Y_{2}}{\partial x_{2}}+\frac{\partial g_{22}}{\partial x_{2}} Y_{2}=0 .
\end{array}
$$

As was have shown in [10], every solution $(g, \Phi)$ of the field equations in (1) gives rise to a corresponding Killing vector field $Y=Y(g, \Phi)$ by way of the local prescription

$$
Y_{i}=\frac{l \epsilon^{i j}}{\sqrt{|\operatorname{det} g|}} \frac{\partial \Phi}{\partial x_{j}}
$$

with $\epsilon^{i j}$ being a permutation symbol. $Y$ preserves both $g$ and $\Phi$. For $g$ in (4) and for the fields $\Phi$ in (5) and (43), the corresponding Killing vector fields are given in (16), (17), and (18) of [11], for example. Our interest of course is in the case of the three solutions $\left(g, \Phi^{(j)}\right)$ in (10) with $g$ given by (6). By (42), $\sqrt{|\operatorname{det} g|}=a^{2} b^{2} \kappa^{2}|\mathrm{sn} \mathrm{cn}| \mathrm{dn}$. Since $Y_{i}$ could be replaced by a scalar multiple of itself (e.g., $-Y_{i}$ ), we shall disregard the absolute value of sn $\mathrm{cn}$ here, and given (9), we shall take $l=$ $b / 2$ (instead of $|b| / 2)$. For $\epsilon^{11}=\epsilon^{22}=0$ and $\epsilon^{12}=-1=-\epsilon^{21}$, (65) then assumes the generic form

$$
\begin{aligned}
& Y_{1}=\left[2 a^{2} b \kappa^{2} \operatorname{sn}(\rho, \kappa) \operatorname{cn}(\rho, \kappa) \operatorname{dn}(\rho, \kappa)\right]^{-1}\left(-\frac{\partial \Phi}{\partial \rho}\right), \\
& Y_{2}=\left[2 a^{2} b \kappa^{2} \operatorname{sn}(\rho, \kappa) \operatorname{cn}(\rho, \kappa) \operatorname{dn}(\rho, \kappa)\right]^{-1} \frac{\partial \Phi}{\partial \tau} ; \\
& Y=Y_{1} \frac{\partial}{\partial \tau}+Y_{2} \frac{\partial}{\partial \rho},
\end{aligned}
$$

where we take $\left(x_{1}, x_{2}\right)=(\tau, \rho)$ in $(64)$.

For the first solution

$$
\Phi^{(1)}(\tau, \rho)=2 a^{2} \operatorname{dn}(\rho, \kappa)+\frac{v^{4}}{4}-a^{2}\left(2-\kappa^{2}\right)
$$

in (10), the computation of the corresponding Killing vector field $Y$ is trivial: by (16) and (66), $Y_{1}=2 / b$, and of course $Y_{2}=0$. Since $\partial g_{11} / \partial \tau=\partial g_{22} / \partial \tau=0$ by (42), the Killing equations in (64) are satisfied and we see that

$$
Y=\frac{2}{b} \frac{\partial}{\partial \tau}
$$

for $\left(g, \Phi^{(1)}\right)$. Computations for the other two solutions $\Phi^{(2)}$ and $\Phi^{(3)}$ in (10) are more involved. The result is the following, where again

$$
A=\frac{1}{16}\left(v^{4}-16 v^{2} a^{2}+8 v^{2} a^{2} \kappa^{2}+16 a^{4} \kappa^{4}\right)
$$

in definition (11). 


$$
\begin{aligned}
& \text { For } \Phi^{(2)}(\tau, \rho), \\
& \qquad \begin{aligned}
Y_{1}= & \frac{(1 / 4)(\sinh (\sqrt{A} \tau))\left[v^{2}+\left(-v^{2} \kappa^{2}-4 a^{2} \kappa^{4}-8 a^{2} \kappa^{2}\right) \operatorname{sn}^{2}(\rho, \kappa)+8 a^{2} \kappa^{4} \operatorname{sn}^{4}(\rho, \kappa)+4 a^{2} \kappa^{2}\right]}{a^{2} b \operatorname{dn}^{2}(\rho, \kappa) \sqrt{v^{2}+\left(-v^{2} \kappa^{2}-4 a^{2} \kappa^{4}\right) \operatorname{sn}^{2}(\rho, \kappa)+4 a^{2} \kappa^{4} \operatorname{sn}^{4}(\rho, \kappa)}} \\
\qquad Y_{2}= & \frac{(\sqrt{A} / 4)(\cosh (\sqrt{A} \tau)) \sqrt{v^{2}+\left(-v^{2} \kappa^{2}-4 a^{2} \kappa^{4}\right) \operatorname{sn}^{2}(\rho, \kappa)+4 a^{2} \kappa^{4} \operatorname{sn}^{4}(\rho, \kappa)}}{a^{2} b \kappa^{2} \operatorname{sn}(\rho, \kappa) \operatorname{cn}(\rho, \kappa) \operatorname{dn}(\rho, \kappa)}
\end{aligned}
\end{aligned}
$$

For $\Phi^{(3)}(\tau, \rho)$ one has quite similar formulas for $Y_{1}$ and $Y_{2}$ except that (as expected) the roles of the hyperbolic sine and hyperbolic cosine in (70) are interchanged: the factor $\sinh (\sqrt{A} \tau)$ for $Y_{1}$ in (70) is replaced by $\cosh (\sqrt{A} \tau)$, and, similarly, $\cosh (\sqrt{A} \tau)$ for $Y_{2}$ in (70) is replaced by $\sinh (\sqrt{A} \tau)$.

One can also find the following alternative expressions for the Killing vector field components for $\Phi^{(2)}(\tau, \rho)$ :

$$
\begin{aligned}
& Y_{1}=\frac{\left(v^{2}+4 a^{2} \kappa^{2} \mathrm{cn}^{2}(\rho, \kappa)-4 a^{2} \kappa^{2} \operatorname{sn}^{2}(\rho, \kappa)\right) \sinh (\sqrt{A} \tau)}{4 a^{2} b \operatorname{dn}(\rho, \kappa) \sqrt{v^{2}-4 a^{2} \kappa^{4} \mathrm{cn}^{2}(\rho, \kappa) \operatorname{sn}^{2}(\rho, \kappa) \mathrm{dn}^{-2}(\rho, \kappa)}}, \\
& Y_{2} \\
& =\frac{4 \sqrt{A} \cosh (\sqrt{A} \tau) \sqrt{v^{2}-4 a^{2} \kappa^{4} \mathrm{cn}^{2}(\rho, \kappa) \operatorname{sn}^{2}(\rho, \kappa) \operatorname{dn}^{-2}(\rho, \kappa)}}{16 a^{2} b \kappa^{2} \operatorname{cn}(\rho, \kappa) \operatorname{sn}(\rho, \kappa)}
\end{aligned}
$$

for $A$ in (11). Corresponding alternative expressions for $\Phi^{(3)}(\tau, \rho)$ are similar to (71) except that the roles of the hyperbolic sine and hyperbolic cosine are interchanged. By a direct check one sees that the dilaton fields computed in (10) are indeed invariant along the corresponding Killing directions. That is, they satisfy

$$
\frac{\partial \Phi^{(i)}}{\partial \tau} Y_{1}+\frac{\partial \Phi^{(i)}}{\partial \rho} Y_{2}=0
$$

for each of $i=1,2,3$ as we indicated in the sentence following (65) about $Y$ preserving $\Phi$.

\section{Some Closing Remarks}

For the metric $g$ in (6), whose derivation was discussed in Section 2, we have obtained as a main result explicit linearly independent solutions $\Phi^{(j)}, j=1,2,3$, in (10) of the corresponding system of dilaton field equations in (9). We have also computed the associated Killing vector fields $Y\left(g, \Phi^{(j)}\right)$ that leave both $g$ and $\Phi^{(j)}$ invariant; see (68), (70), and (71) and the remarks that follow (70) and (71). The dilaton fields simplify to the expressions given in (60) in the special case when the elliptic modulus $\kappa$ is 1 , and $g$ simplifies to the expression given in (61).

For $Q(\rho, \kappa)$ defined in $(24)$, it was shown in the short argument following (22) that if $Q(\rho, \kappa)=0$ for some $\rho$, then necessarily $v^{2} / 4 a^{2} \kappa^{4} \leq 1$ :

$$
|v| \leq 2|a| \kappa^{2}
$$

in contrast to the standing assumption (7). To better understand the meaning of this inequality note first by (42) that $Q(\rho, \kappa)=0 \Rightarrow g_{11}=0$ so $g$ exhibits a horizon singularity at

$$
\begin{aligned}
\frac{a^{2} \kappa^{4} \operatorname{sn}^{2}(\rho, \kappa) \mathrm{cn}^{2}(\rho, \kappa)}{\operatorname{dn}^{2}(\rho, \kappa)} & =\frac{v^{2}}{4}: \\
\frac{\operatorname{sn}(\rho, \kappa) \operatorname{cn}(\rho, \kappa)}{\operatorname{dn}(\rho, \kappa)} & = \pm \frac{v}{2 a \kappa^{2}}
\end{aligned}
$$

again by (24). Keep in mind that $v$ is a velocity parameter of a dissipative soliton (also called a dissipaton) as in (15), for example, especially for $\kappa=1$, as we have remarked at the end of Section 2. Inequality (73) is the statement therefore that for an arbitrary elliptic modulus $\kappa$, with $0<\kappa \leq 1$, the velocity of a black hole dissipaton cannot exceed the limiting value $\left|v_{\text {max }}\right| \stackrel{\text { def. }}{=} 2|a| \kappa^{2}$. This statement was deduced in $[6,7]$, for example, in the special (but important) case of $\kappa=1$.

In Section 3, by a series of explicit transformations of variables, $g$ moreover was transformed to a Jackiw-Teitelboim black hole metric $g_{\mathrm{J}-\mathrm{T}}$ of the simple form (4), namely, to $g_{\mathrm{J}-\mathrm{T}}$ given by (39), with accompanying data given by (40) and (41). Here again assumption (7) was imposed. An advantage of parameterization (39) is that, for example, simple formulas exist $[10,12]$ for thermodynamic quantities such as the Hawking temperature $T_{\mathrm{H}}$ and black hole entropy $S$.

We point out, for the record, that the general solutions of all 2D dilaton gravity models are known. For example, see Section 3 of the paper [13] of Klösch and Strobl. However (again), we have constructed very explicit elliptic solutions that do not follow directly from the results of [13].

Reviewing $[14,15]$, we have added some final remarks that provide a brief review of a connection of the J-T model to cold plasma physics. This connection is facilitated by way of a resonant nonlinear Schrödinger (RNLS) equation.

The authors in [14] consider a system of nonlinear equations that describe the dynamics of two-component cold collisionless plasma in the presence of an external magnetic field B. For uniaxial plasma propagation, this system is reduced to a system that describes the propagation of nonlinear magnetoacoustic waves in cold plasma with a transverse magnetic field. By way of a shallow water approximation of the latter system, a reduction of it to a RNLS equation of the form

$$
i \frac{\partial \psi}{\partial t^{\prime}}+\frac{\partial^{2} \psi}{\partial x^{\prime 2}}-\frac{1}{2}|\psi|^{2} \psi=\left(1+\beta^{2}\right) \frac{1}{|\psi|} \frac{\partial^{2} \psi}{\partial x^{\prime 2}} \psi
$$


is achieved. Here $x^{\prime}=\beta x$ and $t^{\prime}=\beta t$ are rescaled space and time variables, and $\mathbf{B}$ has an expression in terms of a suitable power series expansion in the parameter $\beta^{2}$. $\psi$ has the form $\psi=\sqrt{\rho} e^{-i S}$, where $S\left(x^{\prime}, t^{\prime}\right)$ is a velocity potential and $\rho$ is the mass density of the plasma. Also note the remarks in [15]. A key point of interest for us is that, for

$$
\begin{aligned}
& r \stackrel{\text { def. }}{=} \sqrt{\rho} e^{S / \beta}>0, \\
& s \stackrel{\text { def. }}{=}-\sqrt{\rho} e^{S / \beta}<0, \\
& B \stackrel{\text { def. }}{=} \frac{1}{2 \beta^{2}}=\frac{2}{b^{2}}, \\
& b \stackrel{\text { def. }}{=} 2 \beta,
\end{aligned}
$$

in the variables $\left(x^{\prime}, \tau \stackrel{\text { def. }}{=} \beta t^{\prime}\right)$, the reaction diffusion (RD) system (12) is satisfied; $r$ and $s$ are denoted by $e^{(+)}$and $e^{(-)}$ in [14]. On page 186 of [1], it is shown that, conversely, given solutions $r>0$ and $s<0$ of the RD system (12), one can naturally construct a RNLS solution. By (15), with $b=2 \beta$ by (76), we can take

$$
\begin{gathered}
r\left(x^{\prime}, \tau\right)=2 \alpha \beta \operatorname{dn}\left(a\left(x^{\prime}-v \tau\right), \kappa\right) \\
\cdot \exp \left(\left[\frac{v^{2}}{4}+a^{2}\left(2-\kappa^{2}\right)\right] \tau-\frac{v x^{\prime}}{2}\right), \\
s\left(x^{\prime}, \tau\right)=-2 \alpha \beta \operatorname{dn}\left(a\left(x^{\prime}-v \tau\right), \kappa\right) \\
\cdot \exp \left(-\left[\frac{v^{2}}{4}+a^{2}\left(2-\kappa^{2}\right)\right] \tau+\frac{v x^{\prime}}{2}\right) .
\end{gathered}
$$

All of this means that we can apply prescription (14) to construct a metric $g_{\text {plasma }}$ of constant Ricci scalar curvature $R=4 B \stackrel{\text { def. }}{=} 8 / b^{2} \stackrel{\text { def. }}{=} 2 / \beta^{2}$, as we did in (18), where the notation $t, \rho$ there is now taken to mean $\tau, a\left(x^{\prime}-v \tau\right)$. Moreover, our results show that $g_{\text {plasma }}$ can be transformed to a J-T black hole metric of the form in (4). Thus we can account for a J-T black hole connection in cold plasma physics. Our results also provide elliptic solutions of the corresponding dilaton field equations.

\section{Conflicts of Interest}

The authors declare that there are no conflicts of interest regarding the publication of this paper.

\section{References}

[1] F. Williams, "Some selected thoughts old and new on solitonblack hole connections in $2 \mathrm{~d}$ dilaton gravity," in The SineGordon Model and Its Applications: From Pendula and Josephson Junctions to Gravity and High-Energy Physics, J. CuevasMaraver, P. Kevrekidis, and F. Williams, Eds., pp. 177-204, Springer Publisher, 2014.

[2] R. Jackiw, "A two dimensional model for gravity, in Quantum Theory of Gravity, S. Christensen, Ed., pp. 403-420, Adam Hilger Ltd, 1984.
[3] C. Teitelboim, "The Hamiltonian structure of two-dimensional spacetime and its relation with the conformal anomaly," in Quantum Theory of Gravity, S. Christenson, Ed., pp. 327-344, Adam Hilger Ltd, 1984.

[4] K. Chandrasekharan, Grundlehren der Mathematischen Wissenschaften, vol. 281, Springer-Verlag, 1985.

[5] L. Martina, O. K. Pashaev, and G. Soliani, "Integrable dissipative structures in the gauge theory of gravity," Classical and Quantum Gravity, vol. 14, no. 12, pp. 3179-3186, 1997.

[6] L. Martina, O. K. Pashaev, and G. Soliani, "Bright solitons as black holes," Physical Review D, vol. 58, no. 8, Article ID 084025, 13 pages, 1998.

[7] O. K. Pashaev and J.-H. Lee, "Resonance solitons as black holes in Madelung fluid," Modern Physics Letters A, vol. 17, no. 24, pp. 1601-1619, 2002.

[8] S. Carroll, Spacetime and Geometry: An Introduction to General Relativity, Addison Wesley, 2004.

[9] Y. Matsushima, Differentiable Manifolds, Pure and Applied Mathematics Series, vol. 9, Marcel Dekker, 1972.

[10] J. Gegenberg, G. Kunstatter, and D. Louis-Martinez, "Observables for two-dimensional black holes," Physical Review D, vol. 51, no. 4, pp. 1781-1786, 1995.

[11] J. Gegenberg and G. Kunstatter, "Geometrodynamics of sineGordon solitons," Physical Review D, vol. 58, no. 12, Article ID 124010, 11 pages, 1998.

[12] J. P. Lemos, "Thermodynamics of the two-dimensional black hole in the Teitelboim-Jackiw theory," Physical Review D, vol. 54, no. 10, pp. 6206-6212, 1996.

[13] T. Klösch and T. Strobl, "Classical and quantum gravity in $1+1$ dimensions: I. a unifying approach," Classical and Quantum Gravity, vol. 14, no. 3, p. 825, 1997.

[14] J. H. Lee, O. K. Pashaev, C. Rogers, and W. K. Schief, "The resonant nonlinear Schrödinger equation in cold plasma physics. Application of Bäcklund-Darboux transformations and superposition principles," Journal of Plasma Physics, vol. 73, no. 2, pp. 257-272, 2007.

[15] J.-H. Lee and O. K. Pashaev, "Solitons of the resonant nonlinear Schrödinger equation with nontrivial boundary conditions: Hirota bilinear method," Theoretical and Mathematical Physics, vol. 152, no. 1, pp. 991-1003, 2007. 


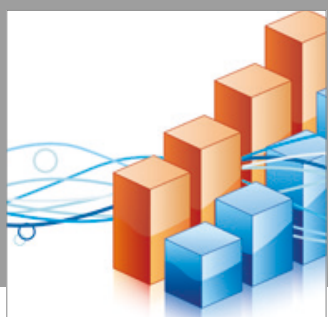

Advances in

Operations Research

vatersals

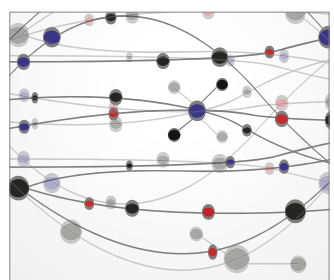

\section{The Scientific} World Journal
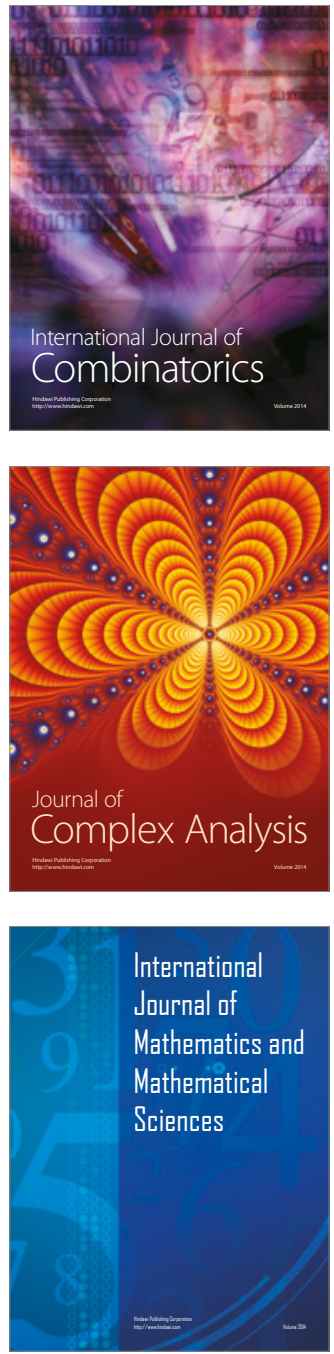
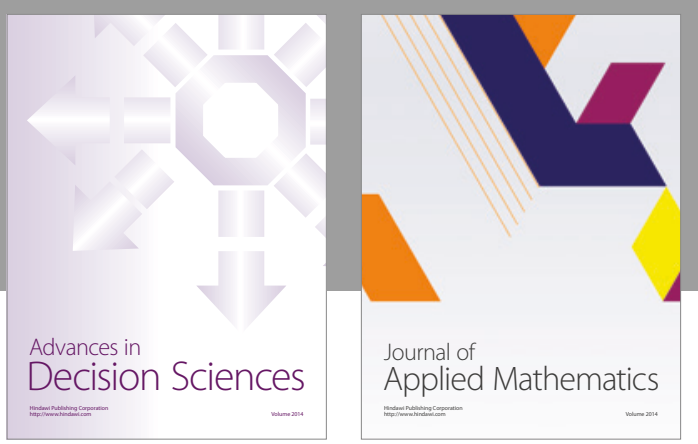

Algebra

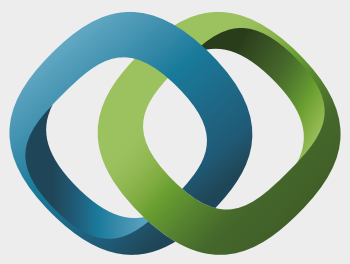

\section{Hindawi}

Submit your manuscripts at

https://www.hindawi.com
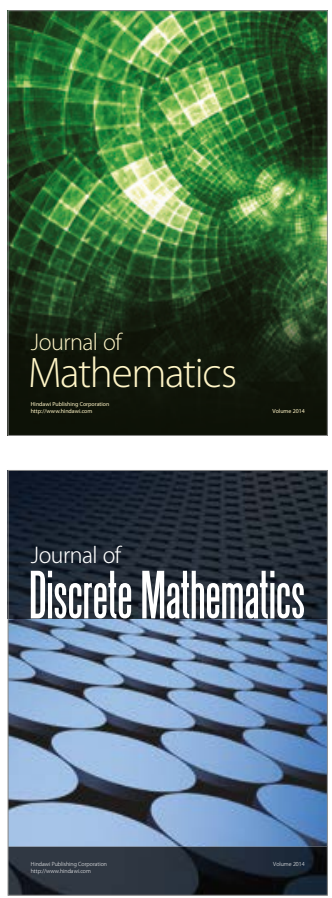

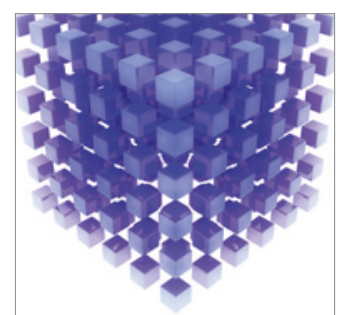

Mathematical Problems in Engineering
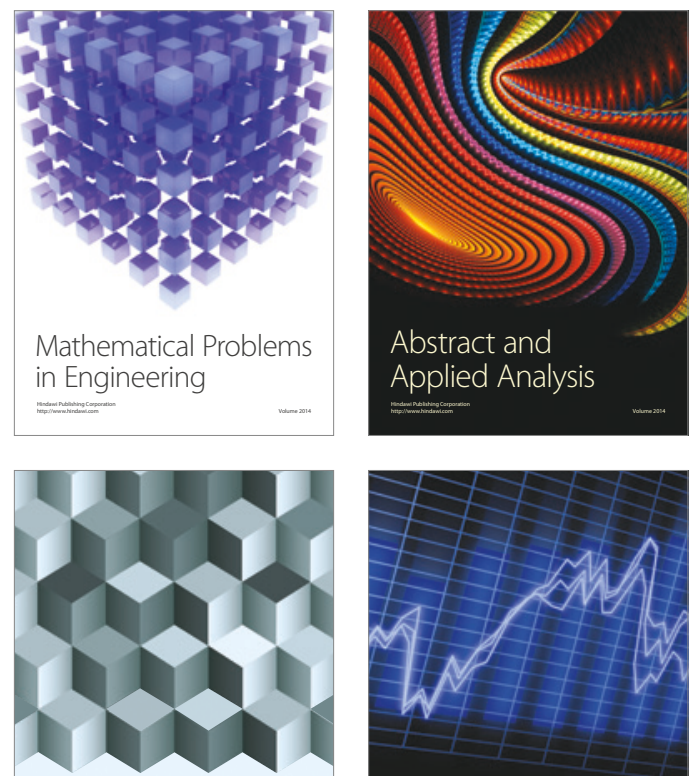

Journal of

Function Spaces

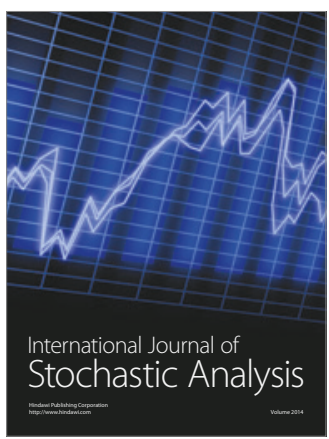

Probability and Statistics
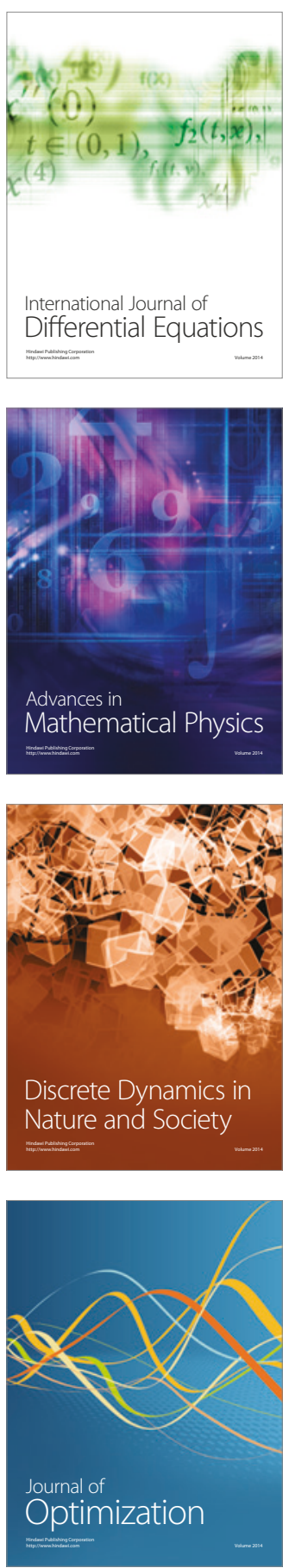